\title{
Epilepsy electroencephalagram singals study based on detrended cross- correlation analysis
}

\author{
Junchang Zhao Wanhu Dou Hongda Ji Jun Wang \\ Image Processing and Image Communications Key Lab, College of Geo \& Bio Infor- \\ mation, Nanjing Univ of Posts \& Telecomm, Nanjing 210003, China \\ wangj@njupt.edu.cn
}

\begin{abstract}
The cross-correlation performance between epilepsy electroencephalogram (EEG) signals reflects the status of epilepsy patients which has importance for analyzing long-range correlation of nonstationary signals. For the first time, detrended cross-correlation analysis (DCCA) was applied to analyze the $\alpha$ wave of different physiological and pathological states of epilepsy EEG signals. It were compared the difference of DCCA values between epilepsy patients' EEG signals and normal subjects' EEG signals. It was found that the DCCA values of epilepsy patients' EEG signals increased compared the normal subjects' EEG signals which can be helpful for medical diagnosis and treatment.
\end{abstract}

Key words: least squares linear fitting, detrended cross-correlation analysis, epilepsy, EEG, $\alpha$ wave

\section{Introduction}

In some cases, the different signals will exhibit a cross-correlation relationship. The presence of these features between signals may be utilized in real life to conduct various analyzes. In the earthquake, the cross-correlation between the degree of noise signals with different detector array antenna can be used as a predictive signal of earthquakes and volcanic eruptions [1]. In business, assets and investment risk estimates are based on the cross-correlation matrix for analysis [2]. In quantum information processing nanotechnology, electronic correlation promoted calculating the cross correlation between the current noise, and were compared to the standard device, and this noise characteristics of the signal can be determined whether it will be inverted [3].

In medicine, it can also be used for diagnosis and treatment of the crosscorrelation characteristics between the signals of different physiological and pathological signals. The paper firstly applied detrende cross-correlation analysis (DCCA) to analyze epilepsy signals, i.e., used least squares curve fitting analysis and detrended covariance to process epilepsy electroencephalogram (EEG) data[4-6]. The DCCA value differences exist between different patients could be shown from the computed results which could guide rehabilitation assessment and treatment. In the same time, it also could contribute to the clinical diagnosis, therapeutic evaluation and study of the mechanism of injury.

Epilepsy is a group of diseases and syndromes, i.e., it is brain sudden abnormal discharge which resulting in a chronic disease of the transient brain dysfunction. Abnormal discharge of neurons in the parts and different range of discharge 
diffusion can be expressed as the onset of movement, feeling the autonomic, consciousness and mental disorders. It is one of the common clinical symptoms caused by a variety of reasons. The human nervous system has complex excited - inhibit the feedback mechanism, in order to maintain the stability of the neuronal membrane. Epilepsy is the enhancement of the excitatory process and weakening of the inhibitory process. Therefore, the analysis of epileptic EEG helps for clinical diagnostics and medical research.

For the first time, detrended crosscorrelation analysis (DCCA) was applied to analyze different physiological and pathological states of epilepsy EEG signals. It were compared the difference of DCCA values between epilepsy patients' EEG signals and normal subjects' EEG signals. It was found that the DCCA values of epilepsy patients' EEG signals increased compared the normal subjects' EEG signals which can be helpful for medical diagnosis and treatment.

When normal adults are in a clear, quiet, eyes closed state, the basic rhythm of the EEG is the occipital $\alpha$ wave while at other sites it is based on a small amount of slow-wave-based $\alpha$ wave. To judge whether brain waves are normal, it is according to their age, the frequency of the EEG, volatility, on both sides of the symmetry and the number of slow-wave part of the way and the presence of pathological wave analysis. It is decisive diagnostic value for epilepsy in Khotan epileptic seizures of intermittent period because the electroencephalogram may have paroxysmal high amplitude slow wave, spikes, sharp wave, spike a slowwave integrated the so-called "painful discharge performance.

In this paper, we used DCCA method to analyze the $\alpha$ wave of different physiological and pathological states of epilepsy EEG signals and reached some inspired results.

\section{Detrended Cross-Correlation Analy-} sis

\subsection{Least Squares Curve Fitting}

The least-squares method uses the extremum method to find the optimal value of the $a$ and $b$ for a set of the measurement data with the given relation. It can make the minimum sum of square for the residuals at each point between the fitted model and the actual observed value. Thereby it can achieve the purpose of the linear fit.

The most commonly used polynomial fitting whose fitting model is,

$$
f(x, A)-a_{0}+a_{1} x+\ldots+a_{n} x^{n}
$$

the coefficient $a_{0}, a_{1}, \ldots, a_{n}$ is determined by the least squares method which is suitable for the case of not the highest power $\mathrm{n}$ polynomial.

When $\mathrm{n}=1$ to obtain a simple fitting model,

$$
y=a+b x
$$

It is called a linear fit which is simple and widely used. Least squares curve fitting in this paper is the simplest linear fit.

\subsection{Detrended Cross-Correlation Anal- ysis}

In order to overcome the highly nonstationary time series, an improved method of analysis of covariance (detrended cross-correlation analysis, DCCA) for cross-correlation analysis of biomedical data.

DCCA is a method based on the detrended covariance analysis. For nonstationary signals, least squares linear fit was made and the trend was eliminated, it can find the cross-correlation between two non-stationary time series.

DCCA provides clinicians with a method studying the long correlation which was generated by internal mechanism of physiological signals. The study 
did not consider the stimulus produced by external system which had no relationship with the system itself. The method used the entire sequence to calculate. So it can provide useful information which can be helpful for doctor diagnosis of a disease.

2 Applying DCCA to epilepsy patients'EEG signals and normal subjects'signals

DCCA was applies into epilepsy patients' and healthy subjects' EEG signals whose results can be helpful for doctor diagnosis of a disease.

Provided that two time series and $\left\{y_{i}^{\prime}\right\}, i=1,2, \ldots, N$.

It was defined that,

$$
\boldsymbol{R}_{k}=y_{1}+y_{2}+\ldots+y_{k}
$$

and

$$
R_{{ }_{k}}^{\prime}=y_{1}^{\prime}+y_{2}^{\prime}+\ldots+y_{k}^{\prime}
$$

Where $k \leq N$.

The mean value were:

$$
\mu=\bar{y}_{i}=(1 / N) \sum_{i=1}^{N} y_{i}
$$

$\mu^{\prime}=\overline{y_{i}^{\prime}}=(1 / N) \sum_{i=1}^{N} y_{i}^{\prime}$

The variance were:

$$
\sigma^{2}=\overline{\left(y_{i}-\overline{y_{i}}\right)^{2}}, \sigma^{\prime 2}=\overline{\left(y_{i}^{\prime}-\overline{y_{i}^{\prime}}\right)^{2}} \text {. }
$$

Assuming the cross-correlation function were : $A(n)=\overline{\left(y_{k}-\mu\right)\left(y_{k+n}-\mu\right)} / \sigma^{2}$

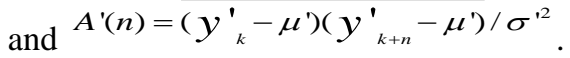
Then the covariance function of the time series can be calculated.

The time sequence was divided into $N-n$ overlaped segments whose each section containing $\boldsymbol{n}+\mathbf{1}$ data, and then each segment was performing a least squares linear fit that was: $R_{k, i}$ and $R_{k, i}^{\prime}$. Each segment's covariance was calculated:

$$
f_{D C C A}^{2}(n, i)=1 /(n-1) \sum_{k=i}^{i+n}\left(R_{k}-R_{k, i}^{\prime}\right)\left(R_{k}^{\prime}-R_{k, i}^{\prime}\right)
$$

At last, the whole time series's covariance was:

$$
F_{D C C A}^{2}(n)=(N-n)^{-1} \sum_{i=1}^{N-n} f_{D C C A}^{2}(n, i)
$$

The above calculation was repeated on segments and then it can be drawn the curve of $F(n)$ and segment size $n$. The slope of $\log (\mathrm{F}(\mathrm{N}))$ vs $\log (\mathrm{n})$ determined the scaling exponent (self-similarity parameter) $\alpha$ [4-6].

3 Results

During the numerical calculation, the EEG were taken from healthy normal

\begin{tabular}{|c|c|c|}
\hline Subjects & Helthy subjects & $\begin{array}{l}\text { Epilepsy sub- } \\
\text { jects }\end{array}$ \\
\hline 1 & 1.673224 & 1.593654 \\
\hline 2 & 1.596377 & 1.575568 \\
\hline 3 & 1.487094 & 1.583341 \\
\hline 4 & 1.588743 & 1.680097 \\
\hline 5 & 1.548715 & 1.582197 \\
\hline 6 & 1.540577 & 1.76196 \\
\hline 7 & 1.569022 & 1.628314 \\
\hline 8 & 1.578676 & 1.603611 \\
\hline 9 & 1.554748 & 1.688994 \\
\hline 10 & 1.520924 & 1.713912 \\
\hline 11 & 1.682928 & 1.98201 \\
\hline 12 & 1.524453 & 1.758955 \\
\hline 13 & 1.5875 & 1.577502 \\
\hline 14 & 1.5334 & 1.63892 \\
\hline 15 & 1.649947 & 1.666177 \\
\hline 16 & 1.569759 & 1.584461 \\
\hline 17 & 1.50896 & 1.587748 \\
\hline 18 & 1.499238 & 1.587887 \\
\hline 19 & 1.669313 & 1.718846 \\
\hline Mean \pm std & $1.5728 \pm 0.0035$ & $1.6586 \pm 0.0101$ \\
\hline
\end{tabular}
people, and people suffering from epilepsy. The two kinds EEG data was analyzed and the results were shown in Table 1 respectively.

Table 1 DCCA of two kinds of subjects'EEG

We can calculated that the $\mathrm{T}$ value is equal to 3.1970 , which means that using DCCA method can distingush the EEG signals from healthy subjects and epilep- 
sy subjects in the confidence probability $\mathrm{P}<0.01$.

It can be seen from the numerical results as shown in Table 1 that the average DCCA value of epilepsy patients' EEG is greater than that of the normal subjects' EEG. It is particularly important to help clinical diagnosis.

Fluctuation range showed the selfsimilar characteristics become unstable when human brain EEG status developing from normal to epilepsy status.

After verification of large amounts of data, it can be achieved a helpful diagnostic method for to make difference between healthy subjects' and epilepsy subjects' EEG. 4 Conclusions

The paper appllied DCCA to analyze the $\alpha$ wave of EEG signals from healthy subjects and epilepsy subjects. It was found that the cross-correlation exists in normal subjects' EEG and the crosscorrelation will decrease for the epilepsy patients' EEG. In clinical applications, doctors can check the DCCA value if it was in the normal EEG' DCCA range to determine if the epilepsy will occur.

\section{Acknowledgements}

This work was supported by the National Natural Science Foundation of China (Grant Nos. 61271082, 61201029, 61102094), the Natural Science Foundation of Jiangsu Province (Grant Nos. BK2011759, BK2011565) and Foundation of Nanjing University of Posts and Telecommunications (JG03212JX02, JG03210JX19, 2011XSG11).

\section{References}

[1] M.Campillo, A.Paul, "Long-range correlations in the diffuse seismic coda," Science, pp. 547-549, 2003.

[2] L.Laloux, P. Cizeau, J.-P. Bouchaud, M. Potters, "Noise dressing of financial correlation matrices," Phys. Rev. Lett., pp.1467-1470, 1999.

[3] P.Samuelsson, E.V. Sukhorukov, M. Buttiker, "Orbital entanglement and violation of bell inequalities in mesoscopic conductors," Phys. Rev. Lett., pp.157002, 2003.

[4] B. Podobnik, H.E. Stanley, "Detrended cross-correlation analysis: A new method for analyzing two nonstationary time series", Phys. Rev. Lett., pp.084102, 2008.

[5] W.-X. Zhou, "Multifractal detrended cross-correlation analysis for two nonstationary signals," Phys. Rev. E, pp.066211, 2008.

[6] P.-A. Absil, R. Sepulchre, A. Bilge, P. Gerard, "Nonlinear analysis of cardiac rhythm fluctuations using DFA method,"Physica A, pp.235244, 1999. 